PROCEEDINGS OF THE

AMERICAN MATHEMATICAL SOCIETY

Volume 127, Number 9, Pages 2573-2581

S 0002-9939(99)04813-3

Article electronically published on May 19, 1999

\title{
TOPOLOGICAL DENSITY OF CCC BOOLEAN ALGEBRAS-EVERY CARDINALITY OCCURS
}

\author{
MARIUSZ RABUS AND SAHARON SHELAH
}

(Communicated by Alan Dow)

\begin{abstract}
For every uncountable cardinal $\mu$ there is a ccc Boolean algebra whose topological density is $\mu$.
\end{abstract}

\section{INTRODUCTION}

For a Boolean algebra $B$, the topological density $d(B)$ is the minimal cardinal $\mu$ such that there is a family $\left\{D_{\xi}: \xi \in \mu\right\}$ of ultrafilters of $B$ with the union $B \backslash\{0\}$. Note that if $S t(B)$ is the Stone space of $B$, then $d(B)$ is the density of $S t(B)$ (as a topological space). A Boolean algebra $B$ has the countable chain condition (ccc) if there is no uncountable collection of pairwise disjoint elements of $B \backslash\{0\}$.

The question we consider in this paper is: what cardinals are topological densities of ccc Boolean algebras? Hajnal, Juhász, and Szentmiklóssy [HJS] prove that under some mild set-theoretic assumptions every uncountable cardinal is the topological density of some ccc Boolean algebra. We prove here that the above statement is a theorem of ZFC.

Theorem 1. For every uncountable cardinal $\mu$ there is a ccc Boolean algebra $\mathcal{B}$, such that $d(\mathcal{B})=\mu$.

The rest of the paper is devoted to the proof of the theorem. Let $\mu$ be an uncountable cardinal. The idea of the proof is to define $\mathcal{B}$ as a quotient of a free Boolean algebra generated by $\left\{x_{\nu}: \nu \in T\right\}$, where $T$ is a set of cardinality $2^{\mu}$. The reason we index the generators by a set $T$, rather than $2^{\mu}$, is that an additional structure on $T$ is helpful in defining the quotient. In particular, the quotient is defined by imposing a set of restrictions of the form $x_{\nu_{0}} \cap x_{\nu_{1}} \cap\left(-\left(x_{\eta_{0}} \Delta x_{\eta_{1}}\right)\right)=0$, for some $\nu_{0}, \nu_{1}, \eta_{0}, \eta_{1}$ in $T$. The definition of $T$ is quite technical but it is the key element of the proof that the topological density of our algebra is $\geq \mu$ (Lemma 4.1).

The motivation behind the construction of $T$ (and getting the density large) is the following observation which we state here, in the countable version, since it may be of more general interest. The property below is actually a little stronger than the one we really use.

Received by the editors January 23, 1997 and, in revised form, October 27, 1997.

1991 Mathematics Subject Classification. Primary 06E15; Secondary 54D80.

Key words and phrases. Topological density, ccc Boolean algebras.

This research was supported by the Basic Research Foundation, administered by the Israeli Academy of Sciences and Humanities. Publication No. 631. 
Claim 1.1. Let $B$ be a Boolean algebra and assume that there is a set $X \subset B$ such that $X$ is not the union of finitely many centered sets and for each $\nu<\omega_{1}$ we have

$$
\begin{aligned}
\forall\left\langle x_{\xi}: \xi<\nu^{+}\right\rangle \subset X \forall F:\left[\nu^{+}\right]^{2} \stackrel{1-1}{\longrightarrow} \nu^{+} \\
\quad \exists\left\langle y_{\xi}: \xi<\nu^{+}\right\rangle \subset X \forall\{\xi, \zeta\} \in\left[\nu^{+}\right]^{2} y_{\xi} \wedge y_{\zeta} \leq x_{F(\xi, \zeta)} .
\end{aligned}
$$

Then the topological density of $B$ is at least $\omega_{1}$.

The construction of $T$ is done in section 2. In section 3 we give the definition of the algebra $\mathcal{B}$ and prove that it has the ccc. In the last section we prove that the topological density of $\mathcal{B}$ is exactly $\mu$.

\section{Preliminaries}

In this section we define the set $T$ and the set of quadruples used in the definition of $\mathcal{B}$. For a cardinal $\sigma<\mu$ let $h_{\sigma}:\left[\sigma^{+}\right]^{2} \rightarrow \sigma^{+}$be such that

(a) it is one-to-one,

(b) for every $X \in\left[\sigma^{+}\right]^{\sigma^{+}}$and $j<\sigma$ there is $j_{1} \in(j, \sigma)$ and $i_{0}, i_{1} \in X$ such that $h_{\sigma}\left(i_{0}, i_{1}\right)=j_{1} \bmod \sigma$.

To prove that such a function exists it is enough to show that there is a function $h_{\sigma}^{\prime}:\left[\sigma^{+}\right]^{2} \rightarrow \sigma$ such that (b) holds. Indeed, then we can define $h_{\sigma}$ to be any 1-1 function from $\sigma^{+}$to $\sigma^{+}$such that $h_{\sigma}(\alpha, \beta)=h_{\sigma}^{\prime}(\alpha, \beta) \bmod \sigma$.

In order to define $h_{\sigma}^{\prime}$, first fix 1-1 functions $q_{\alpha}: \alpha \rightarrow \sigma$ for every $\alpha<\sigma^{+}$. Now for $\alpha<\beta$ define $h_{\sigma}^{\prime}(\alpha, \beta)=q_{\beta}(\alpha)$. To prove (b) let $X \in\left[\sigma^{+}\right]^{\sigma^{+}}$. Let $\beta \in X$ be such that the set $X_{\beta}=\{\alpha \in X: \alpha<\beta\}$ has cardinality $\sigma$. Since $q_{\beta}$ is 1-1 it follows that the image of $\left[X_{\beta}\right]^{2}$ under $h_{\sigma}^{\prime}$ is cofinal in $\sigma$; hence (b) holds.

Definition 2.1. We define, by induction on $\alpha \leq \mu^{+}, \mathcal{T}_{\alpha}=\left(T_{\alpha}, \sigma_{\alpha}, P_{\alpha}^{l}, F_{\alpha}^{m}\right)$ $(l<8, m<2)$ such that:

(1) $T_{\alpha}$ is a set of finite sequences,

(2) $\left\langle P_{\alpha}^{l}: l<8\right\rangle$ is a partition of $T_{\alpha}$,

(3) $\sigma_{\alpha}$ is a function from $T_{\alpha}$ to $\left\{\sigma: \aleph_{0} \leq \sigma \leq \mu\right\}$,

(4) $F_{\alpha}^{m}$ is a partial, two-place, symmetric function from $P_{\alpha}^{7}$ to $T_{\alpha}$,

(5) $\mathcal{T}_{\alpha}$ is increasing, continuous in $\alpha$, i.e., if $\beta<\alpha$, then
(a) $T_{\beta} \subseteq T_{\alpha}$,
(b) $P_{\beta}^{l}=P_{\alpha}^{l} \cap T_{\beta}$ for $l<8$,
(c) $\sigma_{\beta}=\sigma_{\alpha} \uparrow T_{\beta}$,
(d) $F_{\beta}^{m}=F_{\alpha}^{m} \uparrow\left[T_{\beta}\right]^{2}$ for $m<2$,
(e) if $\alpha$ is a limit, then $T_{\alpha}=\bigcup_{\beta<\alpha} T_{\beta}$.

Case 1. $\alpha=0$. Define $T_{0}=\{\langle\rangle\}, P_{0}^{0}=\{\langle\rangle\}$, (so $P_{0}^{l}=\emptyset$ for $\left.l \neq 0\right)$. $\sigma_{0}(\langle\rangle)=\mu$.

Case 2. $\alpha=1$. Define $T_{1}=T_{0} \cup\left\{\langle\sigma\rangle: \aleph_{0} \leq \sigma \leq \mu\right\}, P_{1}^{1}=\left\{\langle\sigma\rangle: \aleph_{0} \leq \sigma \leq \mu\right\}$, $\sigma_{1}(\langle\sigma\rangle)=\sigma$.

Case 3. $\alpha$ is a limit. Put $T_{\alpha}=\bigcup_{\beta<\alpha} T_{\beta}, P_{\alpha}^{l}=\bigcup_{\beta<\alpha} P_{\beta}^{l}, \sigma_{\alpha}=\bigcup_{\beta<\alpha} \sigma_{\beta}$. 
Case 4. $\alpha=\beta+1$. Define $T_{\alpha}=T_{\beta} \cup\left\{T_{\alpha, l}: l<8\right\}$, where

$$
\begin{aligned}
& T_{\alpha, 0}=\emptyset \text {, } \\
& T_{\alpha, 1}=\left\{\eta \curlyvee\langle\sigma\rangle: \eta \in T_{\beta} \backslash\left(P_{\beta}^{5} \cup P_{\beta}^{7}\right), \eta \curlyvee\langle\sigma\rangle \notin T_{\beta}, \aleph_{0} \leq \sigma \leq \sigma_{\beta}(\eta)\right\}, \\
& T_{\alpha, 2}=\left\{\eta \curlyvee\langle 0\rangle: \eta \in T_{\beta} \backslash\left(P_{\beta}^{5} \cup P_{\beta}^{7}\right), \eta \frown\langle 0\rangle \notin T_{\beta}\right\}, \\
& T_{\alpha, 3}=\left\{\eta \frown\langle\rho \curlyvee\langle 0\rangle\rangle: \eta \frown\langle\rho\rangle \in T_{\beta} \backslash\left(P_{\beta}^{5} \cup P_{\beta}^{7}\right), \eta \frown\langle\rho \curlyvee\langle 0\rangle\rangle \notin T_{\beta}\right\}, \\
& T_{\alpha}^{4,5}=\left\{\eta \left\langle\langle\rho\rangle: \eta \in T_{\beta}, \rho\right.\right. \text { a sequence of limit length and } \\
& \left.(\forall \zeta<\lg (\rho))\left(\eta \frown\langle\rho \mid \zeta\rangle \in T_{\beta} \text { but } \eta \frown\langle\rho\rangle \notin T_{\beta}\right)\right\}, \\
& T_{\alpha, 4}=\left\{\eta \curlyvee\langle\rho\rangle \in T_{\alpha}^{4,5}: \lg (\rho)<\sigma_{\beta}(\eta)^{+}\right\}, \\
& T_{\alpha, 5}=T_{\alpha}^{4,5} \backslash T_{\alpha, 4}, \\
& T_{\alpha, 6}=\left\{\eta \curlyvee\left\langle\rho \curlyvee\left\langle\nu_{0}, \nu_{1}\right\rangle\right\rangle: \eta \curlyvee\langle\rho\rangle \in T_{\beta} \backslash\left(P_{\beta}^{5} \cup P_{\beta}^{7}\right),\right. \\
& \text { and } \eta\left\langle\langle\rho\rangle \triangleleft \nu_{l}, l=0,1 \text {, and } \nu_{0}, \nu_{1} \in T_{\beta}, \nu_{0} \neq \nu_{1}\right\} \text {, } \\
& T_{\alpha, 7}=\left\{\eta \curlyvee\langle\rho\rangle \curlyvee\langle i\rangle: \eta \curlyvee\langle\rho\rangle \in P_{\beta}^{5} \text {, and } i<\sigma_{\beta}(\eta)^{+} \text {, and } \eta \curlyvee\langle\rho\rangle\left\langle\langle i\rangle \notin T_{\beta}\right\}\right. \text {. }
\end{aligned}
$$

Let $P_{\alpha}^{l}=P_{\beta}^{l} \cup T_{\alpha, l}$ for $l<8$, and define $\sigma_{\alpha}$ by:

$$
\sigma_{\alpha}(\tau)= \begin{cases}\sigma_{\beta}(\tau) & \text { if } \tau \in T_{\beta}, \\ \tau(n) & \text { if } \tau \in T_{\alpha, 1}, \lg (\tau)=n+1, \\ \sigma_{\beta}(\tau\lceil n) & \text { if } \tau \in\left\{T_{\alpha, l}: l \neq 1\right\}, \lg (\tau)=n+1 .\end{cases}
$$

Finally, define $F_{\alpha}^{m}, m=0,1 . F_{\alpha}^{m}\left(\tau_{1}, \tau_{2}\right)$ is well defined if $F_{\beta}^{m}\left(\tau_{1}, \tau_{2}\right)$ is well defined, or for some $\eta \succ\langle\rho\rangle \in P_{\beta}^{5}$ for $l=1,2$, we have $\tau_{l}=\eta \succ\langle\rho\rangle\left\langle\left\langle i_{l}\right\rangle, i_{1} \neq i_{2}\right.$, and $\rho(\zeta)$ is a pair $\left(\nu_{0}, \nu_{1}\right)$, i.e., $\eta \frown\left\langle\rho \mid \zeta \curlyvee\left\langle\nu_{0}, \nu_{1}\right\rangle\right\rangle \in P_{\alpha}^{6}$, where $\zeta=h_{\sigma_{\beta}(\eta)}\left(i_{1}, i_{2}\right)$. In the first case define $F_{\alpha}^{m}\left(\tau_{1}, \tau_{2}\right)=F_{\beta}^{m}\left(\tau_{1}, \tau_{2}\right)$. In the second case define $F_{\alpha}^{m}\left(\tau_{1}, \tau_{2}\right)=\nu_{m}$.

Proposition 2.2. (1) $T_{\alpha}$ is well defined for $\alpha \leq \mu^{+}$.

(2) Each member of $T_{\alpha}\left(\alpha \leq \mu^{+}\right)$is a finite sequence.

(3) For every $\eta \in T_{\mu^{+}}$, the sequence $\langle\sigma(\eta\lceil k): k \leq \lg (\eta)\rangle$ is non-increasing.

(4) If $\eta\left\langle\rho \rho \in P_{\alpha}^{5}\right.$ and $m_{1}, m_{2}<2, \tau_{1}, \tau_{2}, \tau_{3}, \tau_{4} \in\left\{\eta\left\langle\langle\rho\rangle\left\langle\langle i\rangle: i<\sigma(\eta)^{+}\right\}\right.\right.$ and $F_{\alpha}^{m_{1}}\left(\tau_{1}, \tau_{2}\right)=F_{\alpha}^{m_{2}}\left(\tau_{3}, \tau_{4}\right)$, then $m_{1}=m_{2},\left\{\tau_{1}, \tau_{2}\right\}=\left\{\tau_{3}, \tau_{4}\right\}$. Moreover, the conclusion holds if we assume that $F_{\alpha}^{m_{1}}\left(\tau_{1}, \tau_{2}\right) \uparrow \lg (\eta \frown \rho)=$ $F_{\alpha}^{m_{2}}\left(\tau_{3}, \tau_{4}\right) \uparrow \lg (\eta-\rho)$.

(5) If $\eta \in P_{\mu^{+}}^{7}$, then $\eta$ is maximal in $\left(T_{\mu^{+}}, \triangleleft\right)$, otherwise $\eta$ has infinitely many successors in $T_{\mu^{+}}$

(6) $\left|T_{\mu^{+}}\right|=2^{\mu}$.

Proof. Straightforward.

Let $T=T_{\mu^{+}}, P^{l}=P_{\mu^{+}}^{l}$ for $l<8, \sigma=\sigma_{\mu^{+}}$and $F_{m}=F_{\mu^{+}}^{m}, m=0,1$.

Definition 2.3. (1) We say that $X \subseteq T$ is 1-closed if:

(a) \langle\rangle$\in X$,

(b) if $\eta \triangleleft \eta_{1}, \eta_{1} \in X$, then $\eta \in X$,

(c) if $\eta \succ\langle\rho\rangle \in P^{5}$ and for $k=1,2, \tau_{k}=\eta \succ\langle\rho\rangle\left\langle\left\langle i_{k}\right\rangle \in X, i_{1} \neq i_{2}\right.$ and $m<2$, then $F_{m}\left(\tau_{1}, \tau_{2}\right) \in X$ if it is well defined.

(2) We say that $X \subseteq T$ is 2-closed if it is 1-closed and:

(d) if $\eta\left\langle\left\langle\rho\left\langle\left\langle\nu_{0}, \nu_{1}\right\rangle\right\rangle \in P^{6} \cap X\right.\right.$, then $\nu_{0}, \nu_{1} \in X$, 
(e) if $\eta \curlyvee\left\langle\rho_{1}\right\rangle, \eta\left\langle\rho_{2}\right\rangle \in X$ and $\zeta=\sup \left\{\xi: \rho_{1}\left\lceil\xi=\rho_{2}\lceil\xi\}\right.\right.$, then $\eta \succ\left\langle\rho_{1}\lceil(\zeta+1)\rangle \in X\right.$ if $\zeta<\lg \left(\rho_{1}\right)$, and $\eta \succ\left\langle\rho_{2}\lceil(\zeta+1)\rangle \in X\right.$ if $\zeta<\lg \left(\rho_{2}\right)$.

Proposition 2.4. $\quad$ (1) $T_{\alpha}$ is 2-closed for $\alpha \leq \mu^{+}$.

(2) The family of $k$-closed sets is closed under intersections, $k=1,2$.

(3) If $X \subseteq T$ is finite, then $\operatorname{cl}_{k}(X)$ is finite, $k=1,2$.

Proof. (1) and (2) are straightforward. To prove (3), prove by induction on $\alpha$, that if $X \subseteq T_{\alpha}$ is finite, then $\operatorname{cl}_{k}(X)$ is finite.

\section{Definition of the Algebra, And the CCC}

In this section, we first define the algebra, and second, we prove that it has the ccc. The proof is preceded by two propositions, which give a sufficient condition for an element of the algebra to be non-zero.

Definition 3.1. (1) $\mathcal{B}_{T}$ is the Boolean algebra generated by $\left\{x_{\eta}: \eta \in T\right\}$ freely, except the equations in the following set:

$\Gamma=\left\{\mathbf{e}_{\tau_{1}, \tau_{2}}=\left[x_{\tau_{1}} \cap x_{\tau_{2}} \cap\left(-\left(x_{F_{0}\left(\tau_{1}, \tau_{2}\right)} \Delta x_{F_{1}\left(\tau_{1}, \tau_{2}\right)}\right)\right)=0\right]:\right.$ $\tau_{1}, \tau_{2} \in T$, and $F_{m}\left(\tau_{1}, \tau_{2}\right)$ is well defined, $\left.m=0,1\right\}$.

(2) For $X \subseteq T$ let

$$
\Gamma_{X}=\left\{\mathbf{e}_{\tau_{1}, \tau_{2}}: \tau_{1}, \tau_{2} \in X, \text { and } F_{l}\left(\tau_{1}, \tau_{2}\right) \text { is well defined } l=0,1\right\} .
$$

(3) For $\alpha<\mu$ define $\mathcal{B}_{T_{\alpha}}$ to be the subalgebra of $\mathcal{B}_{T}$ generated by $\left\{x_{\eta}: \eta \in T_{\alpha}\right\}$.

(4) $\mathcal{B}_{0}$ is the trivial Boolean algebra with the universe $\{0,1\}$.

Note: for $\eta \in T$ we consider $x_{\eta}$ to be an element of $\mathcal{B}_{T}$, i.e., it is an equivalence class of the element $x_{\eta}$.

Proposition 3.2. For a Boolean term $\mathbf{t}=t\left(y_{0}, \ldots, y_{n-1}\right)$ and $\eta_{0}, \ldots, \eta_{n-1} \in T$, $\mathcal{B}_{T} \vDash t\left(x_{\eta_{0}}, \ldots, x_{\eta_{n-1}}\right)>0$ if and only if there is a function $f: T \rightarrow\{0,1\}$ such that $\mathcal{B}_{0} \vDash t\left(f\left(\eta_{0}\right), \ldots, f\left(\eta_{n-1}\right)\right)=1$ and $(*)_{f, T}$ holds, where for $X \subseteq T$ we define:

$(*)_{f, X}$ If $\mathbf{e}_{\tau_{1}, \tau_{2}} \in \Gamma_{X}$ and $f\left(\tau_{1}\right)=1=f\left(\tau_{2}\right)$, then $f\left(F_{0}\left(\tau_{1}, \tau_{2}\right)\right) \neq f\left(F_{1}\left(\tau_{1}, \tau_{2}\right)\right)$.

Proof. (1) Assume that $f: T \rightarrow\{0,1\}$ is such that $\mathcal{B}_{0} \vDash t\left(f\left(\eta_{0}\right), \ldots, f\left(\eta_{n-1}\right)\right)=1$ and $(*)_{f, T}$ holds. Note that $\mathcal{B}_{T} \models t\left(x_{\eta_{0}}, \ldots, x_{\eta_{n-1}}\right)>0$ if and only if there is a homomorphism $h: \mathcal{B}_{T} \rightarrow \mathcal{B}_{0}$ such that $\mathcal{B}_{0} \models h\left(t\left(x_{\eta_{0}}, \ldots, x_{\eta_{n-1}}\right)\right)=1$. The function $f: T \rightarrow\{0,1\}$ defines a homomorphism $\bar{f}$ from a free algebra generated by $\left\{x_{\eta}: \eta \in T\right\}$ into $\mathcal{B}_{0}$. Such a homomorphism induces a homomorphism of $\mathcal{B}_{T}$ into $\mathcal{B}_{0}$ if and only if $\bar{f}\left(x_{\tau_{1}} \cap x_{\tau_{2}} \cap\left(-\left(x_{F_{0}\left(\tau_{1}, \tau_{2}\right)} \Delta x_{F_{1}\left(\tau_{1}, \tau_{2}\right)}\right)\right)\right)=0$ for $\tau_{1}, \tau_{2}$ such that $F_{m}\left(\tau_{1}, \tau_{2}\right)$ are well defined. Clearly this is equivalent to $(*)_{f, T}$.

(2) Assume $\mathcal{B}_{T} \vDash t\left(x_{\eta_{0}}, \ldots, x_{\eta_{n-1}}\right)>0$. Without loss of generality

$$
t\left(x_{\eta_{0}}, \ldots, x_{\eta_{n-1}}\right)=\bigcap_{l<n} x_{\eta_{l}}^{\epsilon(l)},
$$

where $\epsilon: n \rightarrow\{0,1\}$, and $x^{1}=x$, and $x^{0}=-x$. Moreover, we can assume that $\left\{\eta_{0}, \ldots, \eta_{n-1}\right\}$ is 1-closed. Define $f: T \rightarrow\{0,1\}$ by: $f\left(\eta_{l}\right)=\epsilon(l)$ for $l<n$, and $f(\rho)=0$ for $\rho \notin\left\{\eta_{0}, \ldots, \eta_{n-1}\right\}$. Clearly $\mathcal{B}_{0} \models t\left(f\left(\eta_{0}\right), \ldots, f\left(\eta_{n-1}\right)\right)=1$ and $(*)_{f, T}$ holds. 
Proposition 3.3. (1) If $X \subseteq T$ is 1-closed, $f: X \rightarrow\{0,1\},(*)_{f, X}$ holds, $\eta_{0}, \ldots, \eta_{n-1} \in X$, and $t\left(y_{0}, \ldots, y_{n-1}\right)$ is a Boolean term such that $\mathcal{B}_{0} \vDash$ $t\left(f\left(\eta_{0}\right), \ldots, f\left(\eta_{n-1}\right)\right)=1$, then $\mathcal{B}_{T} \vDash t\left(x_{\eta_{0}}, \ldots, x_{\eta_{n-1}}\right)>0$.

(2) For $\eta \neq \nu$ in $T, \mathcal{B}_{T} \vDash x_{\eta} \neq x_{\nu}$; moreover, $\mathcal{B}_{T} \vDash\left(x_{\eta} \backslash x_{\nu}\right)>0$.

(3) If $X \subseteq Y \subseteq T, \operatorname{cl}_{1}(X) \subseteq Y, \eta_{0}, \ldots, \eta_{n-1} \in Y, f: Y \rightarrow\{0,1\}, X \subseteq$ $f^{-1}(\{1\}), t\left(y_{0}, \ldots, y_{n-1}\right)$ is a Boolean term such that

$$
\mathcal{B}_{0} \vDash t\left(f\left(\eta_{0}\right), \ldots, f\left(\eta_{n-1}\right)\right)=1,
$$

and $\mathbf{e}_{\tau_{1}, \tau_{2}} \in \Gamma_{X}$ implies that $\left|\left\{F_{0}\left(\tau_{1}, \tau_{2}\right), F_{1}\left(\tau_{1}, \tau_{2}\right)\right\} \cap f^{-1}(\{1\})\right|=1$, then $\mathcal{B}_{T} \vDash t\left(x_{\eta_{0}}, \ldots, x_{\eta_{n-1}}\right)>0$.

Proof. (1) Use 3.2 for $f \cup 0_{T \backslash Y}$. For (2) use part (1) with $X=T, f(\eta)=1$ and $f(\rho)=0$ for $\rho \neq \eta$. (3) is similar to (1).

Lemma 3.4. $\mathcal{B}_{T}$ satisfies the $c c c$, in fact a strong version of the ccc: for every collection of $\kappa=\operatorname{cf}(\kappa)>\aleph_{0}$ elements of $\mathcal{B}_{T}$, there is a subcollection of size $\kappa$ which generates a filter.

Proof. Let $\mathcal{B}_{T} \vDash a_{\alpha}>0$ for $\alpha<\kappa$. Let $a_{\alpha}=t_{\alpha}\left(x_{\eta_{\alpha, 0}}, \ldots, x_{\eta_{\alpha, n_{\alpha}-1}}\right)$, each $t_{\alpha}$ is a Boolean term. Without loss of generality we can assume that:

(1) $\left\{\eta_{\alpha, 0}, \ldots, \eta_{\alpha, n_{\alpha}-1}\right\}$ is 2 -closed for each $\alpha$,

(2) $t_{\alpha}=t, n_{\alpha}=n$, for some $t, n$ and all $\alpha<\kappa$,

(3) $\left\langle\left\{\eta_{\alpha, k}: k<n\right\}: \alpha<\kappa\right\rangle$ is a $\Delta$-system, i.e., for some $n(*) \leq n$ we have: if $k<n(*), \alpha<\kappa$, then $\eta_{\alpha, k}=\eta_{k}$, and $\left\langle\left\{\eta_{\alpha, k}: k \in[n(*), n)\right\}: \alpha<\kappa\right\rangle$ is a sequence of pairwise disjoint sets.

We can assume that $n>n(*)$; otherwise $a_{\alpha}=a_{0}$ for every $\alpha<\kappa$, and we are done. Let $f_{\alpha}: T \rightarrow\{0,1\}$ be such that $(*)_{f_{\alpha}, T}$ holds and

$$
\mathcal{B}_{0} \vDash t\left(f_{\alpha}\left(\eta_{\alpha, 0}\right), \ldots, f_{\alpha}\left(\eta_{\alpha, n-1}\right)\right)=1 .
$$

Without loss of generality we can assume that:

(4) $f_{\alpha}\left(\eta_{\alpha, k}\right)=\mathbf{t}_{k}$, i.e., does not depend on $\alpha$,

(5) the truth values of " $\eta_{\alpha, k_{1}} \triangleleft \eta_{\alpha, k_{2}}$ ", " $\lg \left(\eta_{\alpha, k}\right)=m$ ", " $\eta_{\alpha, k} \in P^{l "}$ do not depend on $\alpha$.

(6) if $\eta \frown\langle\rho\rangle \in P^{5} \cap\left\{\eta_{k}: k<n(*)\right\}, \tau_{1}=\eta \frown\langle\rho\rangle \curlyvee\left\langle i_{1}\right\rangle=\eta_{\alpha, k_{1}}, k_{1} \in[n(*), n)$, and $\tau_{2}=\eta \frown\langle\rho\rangle\left\langle\left\langle i_{2}\right\rangle=\eta_{\alpha, k_{2}}, k_{2} \in[n(*), n), i_{1} \neq i_{2}\right.$, then $F_{0}\left(\tau_{1}, \tau_{2}\right) \notin$ $\left\{\eta_{k}: k<n(*)\right\} ;$ moreover, $F_{0}\left(\tau_{1}, \tau_{2}\right)\left\lceil\lg \left(\eta\langle\rho \rho) \notin\left\{\eta_{k}: k<n(*)\right\}\right.\right.$,

(7) if $\eta \curlyvee\langle\rho\rangle \in P^{5} \cap\left\{\eta_{k}: k<n(*)\right\}, \alpha<\beta<\kappa, \tau_{1}=\eta \curlyvee\langle\rho\rangle\left\langle\left\langle i_{1}\right\rangle=\eta_{\alpha, k_{1}}, k_{1} \in\right.$ $[n(*), n), \tau_{2}=\eta \smile\langle\rho\rangle\left\langle\left\langle i_{2}\right\rangle=\eta_{\beta, k_{2}}, k_{2} \in[n(*), n), i_{1} \neq i_{2}\right.$, then $F_{l}\left(\tau_{1}, \tau_{2}\right) \uparrow$ $\lg \left(\eta\ulcorner\langle\rho\rangle) \notin\left\{\eta_{\alpha, k}: k<n, \alpha<\kappa\right\}\right.$ for $l=0,1$.

It is easy to satisfy $(4)-(6)$. To satisfy $(7)$ note that the function $F(*, *) \uparrow$ $\lg (\eta \frown \rho)$ is $1-1$ by $2.2(4)$. Therefore we can choose a required sequence by induction of length $\kappa$.

Now we will show that if $\alpha_{0}, \ldots, \alpha_{m(*)}<\kappa$, then $\mathcal{B}_{T} \vDash \bigcap_{m \leq m(*)} a_{m}>0$. It is enough to define $f: T \rightarrow\{0,1\}$ such that $(*)_{f, T}$ holds and

$$
\mathcal{B}_{0} \vDash t\left(f\left(\eta_{\alpha_{m}, 0}\right), \ldots, f\left(\eta_{\alpha_{m}, n-1}\right)\right)=1 \text { for } m \leq m(*) .
$$

Define $f(\nu)=1$ if and only if one of the following occurs:

(a) $\nu \in\left\{\eta_{\alpha_{m}, k}: k<n, m \leq m(*)\right\}$ and $f_{\alpha_{m}}(\nu)=1$. 
(b) For some $\eta \frown\langle\rho\rangle \in P^{5} \cap\left\{\eta_{k}: k<n(*)\right\}$ and $m_{1} \neq m_{2}$, and $k_{1}, k_{2} \in$ $[n(*), n)$, we have $\eta \frown\langle\rho\rangle \frown\left\langle i_{1}\right\rangle=\eta_{\alpha_{m_{1}}, k_{1}}, \eta \frown\langle\rho\rangle \frown\left\langle i_{2}\right\rangle=\eta_{\alpha_{m_{2}}, k_{2}}$ and $\nu=$ $F_{0}\left(\eta_{\alpha_{m_{1}}, k_{1}}, \eta_{\alpha_{m_{2}}, k_{2}}\right)$.

Lemma 3.5. (1) $\mathcal{B}_{0} \vDash t\left(f\left(\eta_{\alpha_{m}, 0}\right), \ldots, f\left(\eta_{\alpha_{m}, n-1}\right)\right)=1$ for $m \leq m(*)$.

(2) $(*)_{f, T}$ holds.

Proof. (1) It suffices to prove that $f \uparrow\left\{\eta_{\alpha_{m}, 0}, \ldots, \eta_{\alpha_{m}, n-1}\right\} \subseteq f_{\alpha_{m}}$. Assume first that $f_{\alpha_{m}}\left(\eta_{\alpha_{m}, k}\right)=1$. By the definition of $f$ we have $f\left(\eta_{\alpha_{m}, k}\right)=1$. Now assume that $f\left(\eta_{\alpha_{m}}, k\right)=1$. Hence, one of the cases (a) or (b) holds. If case (a) holds we are done. So suppose that (b) holds. By (7) and the fact each $\left\{\eta_{\alpha, k}: k<n\right\}$ is 2-closed, it follows that $\eta_{\alpha_{m}, k} \notin\left\{\eta_{\alpha, k}: \alpha<\kappa, k<n\right\}$, a contradiction.

(2) Assume that $(*)_{f, T}$ fails. Then there is $\mathbf{e}_{\nu_{1}, \nu_{2}} \in \Gamma_{T}$ such that $\nu_{1}=\eta \frown\langle\rho\rangle \frown\left\langle i_{1}\right\rangle$. $\nu_{2}=\eta \frown\langle\rho\rangle \frown\left\langle i_{2}\right\rangle, i_{1} \neq i_{2}$ and $f\left(\nu_{1}\right)=1=f\left(\nu_{2}\right)$ and $f\left(F_{0}\left(\nu_{1}, \nu_{2}\right)\right)=f\left(F_{1}\left(\nu_{1}, \nu_{2}\right)\right)$. Working toward a contradiction we consider three cases.

Case 1. $\nu_{1}, \nu_{2} \in\left\{\eta_{\alpha_{m}, k}: m \leq m(*), k<n\right\}$. Hence, there is $m_{1}, m_{2} \leq m(*)$, and $k_{1}, k_{2}<n$ such that $\nu_{1}=\eta_{\alpha_{m_{1}}, k_{1}}, \nu_{2}=\eta_{\alpha_{m_{2}}, k_{2}}$.

If $m_{1}=m_{2}$, then as $\left\{\eta_{\alpha_{m_{1}}, k}: k<n\right\}$ is 1-closed, we have $\nu_{1}, \nu_{2}, F_{0}\left(\nu_{1}, \nu_{2}\right)$, $F_{1}\left(\nu_{1}, \nu_{2}\right) \in\left\{\eta_{\alpha_{m_{1}}, k}: k<n\right\}$. Since $(*)_{f_{\alpha_{m_{1}, T}}}$ holds, we get a contradiction.

Hence $m_{1} \neq m_{2}$, and $k_{1}, k_{2} \in[n(*), n)$. By the definition of $f$ we have

$$
f\left(F_{0}\left(\nu_{1}, \nu_{2}\right)\right)=1 \text {, }
$$

so it suffices to show that $f\left(F_{1}\left(\nu_{1}, \nu_{2}\right)\right)=0$. Assume to the contrary that

$$
f\left(F_{1}\left(\nu_{1}, \nu_{2}\right)\right)=1 \text {. }
$$

Hence, as $F_{1}\left(\nu_{1}, \nu_{2}\right) \notin\left\{\eta_{\alpha, k}: \alpha<\kappa, k<n\right\}$ by (7), case (b) must hold. So there is $\eta^{*}\left\langle\rho^{*}\right\rangle \in P^{5} \cap\left\{\eta_{k}: k<n(*)\right\}$ and $\tau_{1}, \tau_{2}$ such that

(i) $\tau_{1}=\eta^{*} \frown\left\langle\rho^{*}\right\rangle \frown\left\langle i_{3}\right\rangle=\eta_{\alpha_{m_{3}}, k_{3}}$,

(ii) $\tau_{2}=\eta^{*} \frown\left\langle\rho^{*}\right\rangle \frown\left\langle i_{4}\right\rangle=\eta_{\alpha_{m_{4}}, k_{4}}$,

(iii) $k_{3}, k_{4} \in[n(*), n)$,

(iv) $m_{3} \neq m_{4}$,

(v) $F_{1}\left(\nu_{1}, \nu_{2}\right)=F_{0}\left(\tau_{1}, \tau_{2}\right)$.

Note that $\eta^{*}\left\langle\rho^{*}\right\rangle \neq \eta \frown\langle\rho\rangle$. On the other hand (v) implies that $\eta=\eta^{*}$ by $2.2(4)$. Let $\zeta=\sup \left\{\xi: \rho^{*} \uparrow \xi=\rho \uparrow \xi\right\}$. Since $\left\{\eta_{k}: k<n(*)\right\}$ is 2-closed, it follows that $\eta \frown\left\langle\rho^{*}\lceil\zeta\rangle=\eta \frown\left\langle\rho\lceil\zeta\rangle \in\left\{\eta_{k}: k<n(*)\right\}\right.\right.$; moreover, both $\eta \mathcal{\gamma}\left\langle\rho^{*} \uparrow(\zeta+1)\right\rangle$ and $\eta \widetilde{\langle\rho}(\zeta+1)\rangle$ are in $\left\{\eta_{k}: k<n(*)\right\}$.

Note that $\lg \left(F_{1}\left(\tau_{1}, \tau_{2}\right)(\lg (\eta \frown\langle\rho\rangle))\right)=\zeta$. Otherwise, $\left\{F_{0}\left(\tau_{1}, \tau_{2}\right), F_{1}\left(\tau_{1}, \tau_{2},\right)\right\} \cap$ $\left\{F_{0}\left(\nu_{1}, \nu_{2}\right), F_{1}\left(\nu_{1}, \nu_{2},\right)\right\}=\emptyset$. Hence, $F_{0}\left(\tau_{1}, \tau_{2}\right) \uparrow \lg (\eta \frown\langle\rho\rangle)=\eta \frown\left\langle\rho\lceil\zeta\rangle \in\left\{\eta_{k}: k<\right.\right.$ $n(*)\}$, contradicting $(7)$.

Case 2. $\left\{\nu_{1}, \nu_{2}\right\} \cap\left\{\eta_{\alpha_{m}, k}: m \leq m(*), k<n\right\}$ is a singleton. By symmetry assume that $\nu_{1} \in\left\{\eta_{\alpha_{m}, k}: m \leq m(*), k<n\right\}$. Hence there are $\eta^{*}\left\langle\rho^{*}\right\rangle \in P^{5} \cap\left\{\eta_{k}: k<\right.$ $n(*)\}, \tau_{1}, \tau_{2}$ and $m \leq m(*), i_{3} \neq i_{4}, k, k_{3}, k_{4}<n$ such that

(a) $\nu_{1}=\eta_{\alpha_{m}, k}$

(b) $\nu_{2}=F_{0}\left(\tau_{1}, \tau_{2}\right)$,

(c) $\tau_{1}=\eta^{*} \frown\left\langle\rho^{*}\right\rangle \frown\left\langle i_{3}\right\rangle$,

(d) $\tau_{2}=\eta^{*} \frown\left\langle\rho^{*}\right\rangle \frown\left\langle i_{4}\right\rangle$. 
It follows that $\eta^{*}\left\langle\rho^{*} \mid \zeta\right\rangle \triangleleft \nu_{2}=\eta\left\langle\langle\rho\rangle\left\langle\left\langle i_{2}\right\rangle\right.\right.$, where $\zeta=h_{\sigma\left(\eta^{*}\right)}\left(i_{3}, i_{4}\right)$.

As the last element of the sequence $\eta^{*}\left\langle\rho^{*} \mid \zeta\right\rangle$ has length $\zeta$ we must have $\eta^{*}\left\langle\rho^{*} \mid \zeta\right\rangle \triangleleft \eta$. Hence, $\eta^{*}\left\langle\rho^{*} \mid \zeta\right\rangle \in\left\{\eta_{\alpha_{m}, k}: k<n\right\}$. Since $\left\{\eta_{\alpha_{m}, k}: k<n\right\}$ is 2closed and it follows by $2.3(2)(\mathrm{e})$ that $\nu_{2} \in\left\{\eta_{\alpha_{m}, k}: k<n\right\}$, we have a contradiction.

Case 3. $\left\{\nu_{1}, \nu_{2}\right\} \cap\left\{\eta_{\alpha_{m}, k}: m<m(*), k<n\right\}=\emptyset$. By the definition there are $\tau_{l}$, $m_{l}, k_{l}, i_{l}$ for $l=1, \ldots, 4$ such that $\nu_{1}=F_{0}\left(\tau_{1}, \tau_{2}\right)$, and $\nu_{2}=F_{0}\left(\tau_{3}, \tau_{4}\right)$, and

(a) $\tau_{1}=\eta_{1}\left\langle\rho_{1}\right\rangle \frown\left\langle i_{1}\right\rangle=\eta_{\alpha_{m_{1}, k_{1}}}, \tau_{2}=\eta_{1}\left\langle\rho_{1}\right\rangle \curlyvee\left\langle i_{2}\right\rangle=\eta_{\alpha_{m_{2}, k_{2}}}$,

(b) $m_{1} \neq m_{2}, i_{1} \neq i_{2}$,

(c) $\tau_{3}=\eta_{2}\left\langle\rho_{2}\right\rangle \smile\left\langle i_{3}\right\rangle=\eta_{\alpha_{m_{3}, k_{3}}}, \tau_{4}=\eta_{2}\left\langle\rho_{2}\right\rangle \smile\left\langle i_{4}\right\rangle=\eta_{\alpha_{m_{4}, k_{4}}}$,

(d) $m_{3} \neq m_{4}, i_{3} \neq i_{4}$,

(e) $\eta_{1}\left\langle\rho_{1}\right\rangle, \eta_{2}\left\langle\rho_{2}\right\rangle \in P^{5} \cap\left\{\eta_{k}: k \leq n(*)\right\}$.

Let $\zeta_{1}=h_{\sigma\left(\eta_{1}\right)}\left(i_{1}, i_{2}\right), \zeta_{2}=h_{\sigma\left(\eta_{2}\right)}\left(i_{3}, i_{4}\right)$. Note that $\eta_{1}\left\langle\rho_{1}\left\lceil\zeta_{1}\right\rangle \triangleleft \eta\langle\langle\rho\rangle\right.$ and $\eta_{2}\left\langle\rho_{2}\left\lceil\zeta_{2}\right\rangle \triangleleft \eta \smile\langle\rho\rangle\right.$. Hence, either $\eta_{1}\left\langle\rho_{1}\left\lceil\zeta_{1}\right\rangle \triangleleft \eta_{2}\left\langle\rho_{2}\left\lceil\zeta_{2}\right\rangle\right.\right.$ or $\eta_{2}\left\langle\rho_{2}\left\lceil\zeta_{2}\right\rangle \triangleleft\right.$ $\eta_{1}\left\langle\rho_{1} \mid \zeta_{1}\right\rangle$. Assume the first case, the other is symmetric. If $\eta_{1}\left\langle\rho_{1} \mid \zeta_{1}\right\rangle=$ $\eta_{2}\left\langle\rho_{2}\left\lceil\zeta_{2}\right\rangle\right.$, then $\zeta_{1}=\zeta_{2}=\sup \left\{\xi: \rho_{1}\left\lceil\xi=\rho_{2}\lceil\xi\}\right.\right.$, as $\nu_{1} \neq \nu_{2}$. Hence, both $\eta_{1}\left\langle\rho_{1}\left\lceil\zeta_{1}\right\rangle\right.$ and $\eta_{2}\left\langle\rho_{2}\left\lceil\zeta_{2}\right\rangle\right.$ are in $\left\{\eta_{k}: k<n(*)\right\}$, and by $2.3 \nu_{1}, \nu_{2} \in\left\{\eta_{k}: k<\right.$ $n(*)\}$, contradiction. Therefore $\eta_{1}\left\langle\rho_{1}\left\lceil\zeta_{1}\right\rangle \triangleleft \eta_{2}\right.$. Since $\eta_{2} \in\left\{\eta_{k}: k<n(*)\right\}$, also $\eta_{1}\left\langle\rho_{1}\left\lceil\zeta_{1}\right\rangle \in\left\{\eta_{k}: k<n(*)\right\}\right.$. As above this implies that $\nu_{1} \in\left\{\eta_{k}: k<n(*)\right\}$, contradiction.

\section{THE EXACT DENSITY}

In this section we prove that the topological density of the algebra $\mathcal{B}$ is $\mu$.

Lemma 4.1. $d\left(\mathcal{B}_{T}\right) \geq \mu$.

Proof. Assume that $d\left(\mathcal{B}_{T}\right)<\mu$. Hence, there is a sequence $\mathcal{D}^{\otimes}=\left\langle D_{j}^{\otimes}: j<d\left(\mathcal{B}_{T}\right)\right\rangle$ of ultrafilters of $\mathcal{B}_{T}$ such that for every $a \in \mathcal{B}_{T} \backslash\{0\}$ there is $j$ such that $a \in D_{j}^{\otimes}$. Let

$$
\begin{aligned}
& \mathcal{F}=\{\sigma: \sigma<\mu \text { and there are } \eta \text { and } \overline{\mathcal{D}} \text { such that: } \\
& \text { (a) } \eta \in T \backslash P^{7}, \sigma(\eta) \geq \sigma, \\
& \text { (b) } \overline{\mathcal{D}}=\left\langle D_{j}: j<\sigma\right\rangle \text { is a sequence of ultrafilters of } \mathcal{B}_{T}, \\
& \text { (c) if } \left.\eta \triangleleft \nu_{1}, \eta \triangleleft \nu_{2}, \nu_{1} \neq \nu_{2} \text {, then } x_{\nu_{1}} \triangle x_{\nu_{2}} \in \bigcup_{j<\sigma} D_{j}\right\} .
\end{aligned}
$$

Note that $\mathcal{F} \neq \emptyset$. In particular \langle\rangle$, \mathcal{D}^{\otimes}$ witness that $d\left(\mathcal{B}_{T}\right) \in \mathcal{F}$. Let $\sigma^{*}=\min (\mathcal{F})$ and let $\eta^{*}, \mathcal{D}^{*}$ witness that $\sigma^{*} \in \mathcal{F}$. Note that $\sigma^{*} \geq \aleph_{0}$. Without loss of generality $\sigma^{*}=\sigma\left(\eta^{*}\right)$, (otherwise use $\eta^{*}\left\{\left\langle\sigma^{*}\right\rangle\right.$ instead of $\sigma^{*}$ ).

Now we choose by induction on $\zeta<\left(\sigma^{*}\right)^{+}$, a sequence $\rho_{\zeta}$ such that:

(1) $\eta^{*}\left\langle\rho_{\zeta}\right\rangle \in T$,

(2) $\lg \left(\rho_{\zeta}\right)=1+\zeta$,

(3) $\xi<\zeta \Longrightarrow \rho_{\xi}=\rho_{\zeta}\lceil(1+\xi)$,

(4) $\eta^{*}\left\langle\rho_{\zeta+1}\right\rangle \in P^{6}, \rho_{\zeta+1}(\zeta)=\left(\nu_{0, \zeta}, \nu_{1, \zeta}\right)$,

(5) if $\zeta=\sigma^{*} \xi+j, j<\sigma^{*}$, then $\left(x_{\nu_{0, \zeta}} \Delta x_{\nu_{1, \zeta}}\right) \notin \bigcup_{i<j} D_{i}^{*}$.

For $\zeta=0$, let $\rho_{0}=\left\langle\sigma^{*}\right\rangle$. For $\zeta$ limit put $\rho_{\zeta}=\bigcup_{\xi<\zeta} \rho_{\xi}$. For $\zeta=\xi+1, \zeta=\sigma^{*} \xi+j$, if we cannot find suitable $\left(\nu_{0}, \nu_{1}\right)$, then $\eta^{*}\left\langle\rho_{\xi}\right\rangle,\left\langle D_{i}^{*}: i<j\right\rangle$ witness that $j \in \mathcal{F}$, contradicting the minimality of $\sigma^{*}$. 


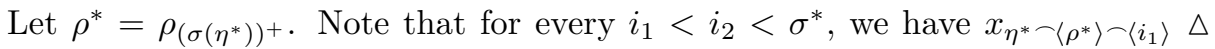
$x_{\eta^{*}-\left\langle\rho^{*}\right\rangle-\left\langle i_{2}\right\rangle} \in \bigcup_{j<\sigma^{*}} D_{j}^{*}$. Hence, there is $j(*)<\sigma^{*}$ such that $X=\left\{i<\left(\sigma^{*}\right)^{+}\right.$: $\left.x_{\eta^{*}-\left\langle\rho^{*}\right\rangle-\langle i\rangle} \in D_{j(*)}^{*}\right\}$ has cardinality $\left(\sigma^{*}\right)^{+}$. Now let $j_{1} \in\left(j(*), \sigma^{*}\right)$ and $i_{0}, i_{1} \in X$ be such that $h_{\sigma^{*}}\left(\left\{i_{0}, i_{1}\right\}\right)=j_{1} \bmod \sigma^{*}\left(j_{1}\right.$ exists by the definition of $\left.h_{\sigma^{*}}\right)$.

Now let $\tau_{0}=\eta^{*}\left\langle\rho^{*}\right\rangle\left\langle\left\langle i_{0}\right\rangle, \tau_{1}=\eta^{*}\left\langle\rho^{*}\right\rangle \curlyvee\left\langle i_{1}\right\rangle\right.$. Hence, $F_{0}\left(\tau_{0}, \tau_{1}\right)=\nu_{0, \epsilon}$ and $F_{1}\left(\tau_{0}, \tau_{1}\right)=\nu_{1, \epsilon}$, where $\epsilon=h_{\sigma^{*}}\left(\left\{i_{0}, i_{1}\right\}\right)$. So $\mathbf{e}_{\tau_{0}, \tau_{1}} \in \Gamma_{T}$. Note that $x_{\tau_{0}} \cap x_{\tau_{1}} \in$ $D_{j(*)}^{*}$, and $x_{\nu_{0, \epsilon}} \triangle x_{\nu_{1, \epsilon}} \notin D_{j_{1}}^{*}$, as $j(*)<j_{1}$. Hence, $-\left(x_{\nu_{0, \epsilon}} \Delta x_{\nu_{1, \epsilon}}\right) \in D_{j(*)}^{*}$. On the other hand, $\mathbf{e}_{\tau_{0}, \tau_{1}} \in \Gamma_{T}$ implies that $x_{\tau_{0}} \cap x_{\tau_{1}} \cap-\left(x_{\nu_{0, \epsilon}} \Delta x_{\nu_{1, \epsilon}}\right)=0$, contradiction.

Lemma 4.2. $d\left(\mathcal{B}_{T}\right) \leq \mu$.

Proof. The idea of the proof is to define a set $\mathcal{F} \subseteq\{1,-1\}^{T}$ such that:

(1) $|\mathcal{F}|=\mu$.

(2) For every $f \in \mathcal{F}$, the set $D_{f}=\left\{x_{\eta}^{f(\eta)}: \eta \in T\right\}$ generates an ultrafilter in $\mathcal{B}_{T}$, where $x^{1}=x$ and $x^{-1}$ is the complement of $x$.

(3) For every $a \in \mathcal{B}_{T}$, non-zero, there is $f \in \mathcal{F}$ such that $a$ is in the ultrafilter generated by $D_{f}$.

First, divide $T$ into three disjoint sets $T=T_{0} \cup T_{1} \cup T_{2}$ as follows. $T_{0}=$ $\bigcup\left\{\left\{\tau_{0}, \tau_{1}\right\}:\left\{\tau_{0}, \tau_{1}\right\} \in \operatorname{dom}\left(F^{i}\right), i=0,1\right\}$. Let $T_{1}$ be the image of $T_{0}$ under $F^{0}$ and $F^{1}$. It follows from the construction of $T$ that $T_{0}$ is disjoint from $T_{1}$. Finally let $T_{2}=T \backslash\left(T_{0} \cup T_{1}\right)$.

Let $F_{2} \subseteq\{1,-1\}^{T_{2}}$ be a set of cardinality $\mu$ such that

(a) for every finite $u \subseteq T_{2}$ and a function $h: u \rightarrow\{1,-1\}$ there is $f \in F_{2}$ such that $h \subseteq f$.

Similarly, let $F_{1} \subseteq\{1,-1\}^{T_{1}}$ be a set of cardinality $\mu$ such that

(b) for every finite $u \subseteq T_{1}$ and a function $h: u \rightarrow\{1,-1\}$ there is $f \in F_{1}$ such that $h \subseteq f$.

Now, for every $f \in F_{1}$ we choose a set $F_{0}^{f} \subseteq\{1,-1\}^{T_{0}}$ of cardinality $\mu$ such that

(c) for every $g \in F_{0}^{f}$, for every $\nu_{0}, \nu_{1} \in T_{1}, \tau_{0}, \tau_{1} \in T_{0}$, if $F^{i}\left(\tau_{0}, \tau_{1}\right)=\nu_{i}$, $i=0,1$ and $f\left(\nu_{0}\right)=f\left(\nu_{1}\right)$, then $\left(g\left(\tau_{0}\right), g\left(\tau_{1}\right)\right) \neq(1,1)$.

(d) $F_{0}^{f}$ is dense with respect to (c), i.e., for every finite $u \subseteq T_{0}$ and a function $h: u \rightarrow\{1,-1\}$ such that the condition (c) is satisfied with $h$ in place of $g$, then there is $g \in F_{0}^{f}$ such that $h \subseteq g$, i.e., $(*)_{f \cup g, T}$ holds.

We have to show that we can choose $F_{0}^{f}$ as required. Let $E=\left\{\left\{\tau_{0}, \tau_{1}\right\} \in\right.$ $\left.\left[T_{0}\right]^{2}: f\left(F_{0}\left(\tau_{0}, \tau_{1}\right)\right) \neq f\left(F_{1}\left(\tau_{0}, \tau_{1}\right)\right)\right\}$. Note that for any function $g \in F_{0}^{f}$ we must have $\left(g\left(\tau_{0}\right), g\left(\tau_{1}\right)\right) \neq(1,1)$. Recall that for any $\left\{\tau_{0}, \tau_{1}\right\},\left\{\tau_{2}, \tau_{3}\right\}$ in $\operatorname{dom}\left(F_{i}\right)$, either $\left\{\tau_{0}, \tau_{1}\right\}=\left\{\tau_{2}, \tau_{3}\right\}$ or $\left\{\tau_{0}, \tau_{1}\right\} \cap\left\{\tau_{2}, \tau_{3}\right\}=\emptyset$. Let $Y_{0}^{f}$ be a dense subset of $\{-1,1\}^{\left(T_{0} \backslash \cup E\right)} \times 3^{E}$ of cardinality $\mu$. For any $h \in 3^{E}$ define $\Phi(h) \in\{1,-1\} \cup E$ as follows: for $\left\{\tau_{0}, \tau_{1}\right\} \in E$ :

if $h\left(\left\{\tau_{0}, \tau_{1}\right\}\right)=0$, then $\Phi(h)\left(\tau_{0}\right)=-1$ and $\Phi(h)\left(\tau_{1}\right)=-1$;

if $h\left(\left\{\tau_{0}, \tau_{1}\right\}\right)=1$, then $\Phi(h)\left(\tau_{0}\right)=1$ and $\Phi(h)\left(\tau_{1}\right)=-1$;

if $h\left(\left\{\tau_{0}, \tau_{1}\right\}\right)=2$, then $\Phi(h)\left(\tau_{0}\right)=-1$ and $\Phi(h)\left(\tau_{1}\right)=1$.

Finally, let $X_{0}^{f}=\left\{g_{0} \cup \Phi\left(g_{1}\right):\left(g_{0}, g_{1}\right) \in Y_{0}^{f}\right\}$. Define $\mathcal{F}=\left\{f_{2} \cup f_{1} \cup f_{0}: f_{2} \in\right.$ $\left.F_{2}, f_{1} \in F_{1}, f_{0} \in F_{0}^{f_{1}}\right\}$.

We prove that $\mathcal{F}$ is as required. It is obvious that (1) holds, and (2) follows from the definition of $\mathcal{F}, D_{f}$ and Proposition 3.2. To prove (3) let $\mathcal{B}_{T}=a>0$. Without 
loss of generality $a=\bigcap_{\eta \in u} x_{\eta}^{h(\eta)}$ for some finite $u \subseteq T$ and $h: u \rightarrow\{1,-1\}$. Let $u_{i}=u \cap T_{i}$ for $i \leq 2$. Let $f_{2} \in F_{2}$ and $f_{1} \in F_{1}$ be such that $h\left\lceil u_{i} \subseteq f_{i}, i=1,2\right.$. Let $f_{0} \in F_{0}^{f_{1}}$ be such that $h\left\lceil u_{0} \subseteq f_{0}\right.$. We have to show that $f_{0}$ exists. Note that $(*)_{h, T}$ holds since $a$ is non-zero; hence $f_{0}$ exists by (d). It follows that $a \in D_{f}$, where $f=f_{2} \cup f_{1} \cup f_{0}$.

This finishes the proof of the theorem.

\section{REFERENCES}

[HJS] A. Hajnal, I. Juhász, Z. Szentmiklóssy, Compact ccc spaces of prescribed density via hypergraphs, Combinatorics, Paul Erdös is eighty, Vo1. 1, 239-252, Bolyai Soc. Math. Stud., Budapest, 1993. MR 94i:54009

Department of of Mathematics, York University, North York, Ontario, Canada M3J 1P3

E-mail address: rabus@mathstat.yorku.ca

Institute of Mathematics, The Hebrew University of Jerusalem, Jerusalem 91904, ISRAEL

E-mail address: shelah@math.huji.ac.il 